\title{
Preeclampsia Is Associated with Abnormal Expression of Adhesion Molecules by Invasive Cytotrophoblasts
}

\author{
Yan Zhou, * Caroline H. Damsky, ${ }^{* \|}$ King Chiu, * James M. Roberts, ${ }^{\star}$ and Susan J. Fisher *\$\$ \\ Departments of *Stomatology, ${ }^{\ddagger}$ Obstetrics, Gynecology, and Reproductive Sciences, ${ }^{\S}$ Pharmaceutical Chemistry and $"$ Anatomy, \\ University of California San Francisco, San Francisco, California 94143
}

\begin{abstract}
In normal human pregnancy, invasion of the uterus and its arterial system by cytotrophoblasts extends through the entire decidua and the adjacent third of the myometrium. Our previous work showed that during the first trimester of pregnancy, invasion is accompanied by a marked change in the expression of cell adhesion molecules by invasive cytotrophoblasts. In the pregnancy disorder preeclampsia, cytotrophoblast invasion is limited to the superficial decidua, and few arterioles are breached. The purpose of this study was to determine whether cytotrophoblast expression of adhesion molecules in this disorder is also abnormal. Placental bed biopsy specimens from normal pregnancies and those complicated by preeclampsia were stained with anti-integrin antibodies. The results showed that adhesion molecule switching by invasive cytotrophoblasts is abnormal in preeclampsia, which suggests that this subpopulation of trophoblast cells fails to differentiate properly. A likely result is that the delicate balance of adhesive interactions that normally permit cytotrophoblast invasion is tipped in favor of those which restrain this process, with the net effect of shallow uterine invasion. (J. Clin. Invest. 1993. 91:950-960.) Key words: placenta • differentiation • integrin • uterus • immunocytochemistry
\end{abstract}

\section{Introduction}

Preeclampsia, which most commonly develops in primagravidas, is variously estimated to affect $7-10 \%$ of all pregnancies (for review see reference 1 ). The signs, including maternal increased blood pressure and renal dysfunction evidenced by proteinuria and edema, usually develop in the late second or third trimester. In addition to these effects on the mother, there is a profound impact on the fetus, resulting in increased perinatal mortality and frequent intrauterine growth retardation. There is no cure for this disease other than termination of pregnancy. When the maternal or fetal condition mandates expeditious delivery, iatrogenic prematurity is often the result.

Despite the widespread occurrence of preeclampsia, the underlying cause or causes of this disease remain elusive. However, extensive studies of the pathophysiology of this syndrome provide insights into its etiology. The accumulated evidence

Address correspondence to Susan Fisher, HSW 604, University of California, San Francisco, San Francisco, CA 94143-0512.

Received for publication 22 June 1992 and in revised form 10 September 1992.

J. Clin. Invest.

(c) The American Society for Clinical Investigation, Inc.

$0021-9738 / 93 / 03 / 0950 / 11 \quad \$ 2.00$

Volume 91, March 1993, 950-960 strongly suggests that the causative agent is the placenta $(2,3)$. For example, preeclampsia can occur in hydatidiform mole (4), where no fetus is present.

Since trophoblast invasion is abnormally shallow in preeclampsia, it is likely that some component of the invasion process is altered. Normally, cytotrophoblasts from anchoring villi invade the uterine parenchyma and its associated arteries to the level of the inner third of the myometrium (5-7). The vascular component of the invasion process gives rise to "physiologic changes," a term used to describe the loss of the endothelial lining and most of the musculoelastic tissue of the spiral arteries, with a striking increase in the caliber of these vessels (8). These changes occur in preparation for the dramatic increase in blood flow demand the fetus places on the mother.

Several morphological studies have described the faulty trophoblast invasion associated with preeclampsia. Two types of abnormalities can be seen in the same specimen. First, trophoblast invasion of the uterine parenchyma is uniformly shallow, and invasion of the vasculature does not proceed beyond the decidual portions of the spiral arteries. Thus, the maternal vessels do not undergo the complete spectrum of physiologic changes. As a result, the mean external diameter of the myometrial vessels is less than half that of similar vessels from normal pregnancies (9-11). Second, the number of vessels that show evidence of trophoblast invasion is decreased (12). These findings are of particular interest since they could produce the reduction in uteroplacental blood flow observed in preeclampsia (13).

Elucidating the exact role of the placenta in the development of preeclampsia has been hampered by the fact that fundamental aspects of the biology of this organ are not well understood. Our laboratory has been studying mechanisms used by human cytotrophoblasts to invade the uterus in normal pregnancy. Recently, we used tissue sections of the human placental bed to show that the staining pattern of several adhesion molecules undergoes a dramatic alteration during normal cytotrophoblast differentiation along the invasive pathway in vivo (7). This and subsequent in vitro studies (reference 14 and Damsky, C. H., and S. J. Fisher, manuscript in preparation) suggest that these alterations in cytotrophoblast cell-cell and cell-extracellular matrix interactions are likely to have important functional significance with regard to the invasion process. For this reason we initiated the following study in which we asked whether the abnormally shallow invasion that is characteristic of preeclampsia is associated with abnormal cytotrophoblast expression of adhesion molecules.

\section{Methods}

Patient selection. Women who received prenatal care at the University of California San Francisco (Moffitt/Long Hospital, San Francisco General Hospital) and who had no underlying medical conditions were 
enrolled in this study. During one of the late second trimester/early third trimester clinic visits, informed consent was obtained to remove a placental bed biopsy specimen if a cesarean section was performed. The consent form and procedure for performing the biopsy were approved by the University of California San Francisco Committee on Human Research.

Samples of floating chorionic villi, basal plate, and the placental bed, all from the same pregnancy, were obtained during cesearean section from both normal patients and those with preeclampsia. These samples, which were collected by direct visualization of the placental site, were studied in great detail and form the basis of this report. Normal patients were normotensive throughout pregnancy and had no proteinuria or other signs of preeclampsia. Eight normal samples were obtained from women who were between 22 wk of gestation and term. Of these, one 22-wk sample was taken from a nulliparous woman who had a previous history of cervical cancer, whose disease recurred locally during pregnancy and who elected to have a late second trimester hysterectomy. Two other samples of floating villi and extensive regions of the basal plate were obtained from nulliparous women who underwent elective terminations, by dilatation and uterine evacuation, at $22 \mathrm{wk}$. Five placental bed specimens were obtained from normal, nulliparous women who underwent cesearean sections at 33 to $40 \mathrm{wk}$ for obstetrical indications. The findings of studies of these placental and placental bed tissues were corroborated in nine additional samples of floating chorionic villi and basal plates obtained by dissection of placentas obtained from normal pregnancies after vaginal deliveries (32-40 wk).

Preeclamptic patients fulfilled the following criteria, recommended by Chesley (15): nulliparity; no history of hypertension before pregnancy; increase in diastolic pressure of $15 \mathrm{mmHg}$ or systolic pressure of $30 \mathrm{mmHg}$ compared with blood pressure obtained before $20 \mathrm{wk}$ of gestation; proteinuria $\geq 0.5 \mathrm{~g} / 24 \mathrm{~h}$ or $\geq 30 \mathrm{mg} / \mathrm{dl}$ in a catheterized specimen; hyperuricemia $>5.5 \mathrm{mg} / \mathrm{dl}$ (or one SD greater than the normal mean value before term; reference 16); return to normal blood pressure and resolution of proteinuria by $12 \mathrm{wk}$ postpartum. Nine samples taken between 25 and 37 wk of gestation were obtained from pregnancies complicated by preeclampsia. The findings of studies of these placental and placental bed tissues were corroborated in six additional samples of floating villi and basal plates obtained by dissection of placentas obtained after vaginal delivery of preeclamptic patients ( 30 $38 \mathrm{wk}$ ).

Antibodies, tissue fixation, and immunocytochemistry. The antibodies, tissue preparation, and specific methods, including cytokeratin staining to identify trophoblasts, were described previously $(7,17)$.

\section{Results}

Comparison of trophoblast morphology in normal and preeclamptic pregnancies. The differences in the morphology of floating villi from normal and preeclamptic pregnancies were consistent with those that have been reported previously (18). The most striking difference was the presence of knob-like protrusions, termed syncytial sprouts or knots, in the floating villi of five of the nine preeclamptic patients. However, these were primarily composed not of multinucleate syncytium but of mononuclear cells that stained for E cadherin (17) and the integrin $\alpha 6$ subunit (7), which within the placenta are cytotrophoblast specific. As suggested previously $(18,19)$, these structures may be evidence of cytotrophoblast proliferation in response to hypoxia.

With regard to extravillus trophoblasts, we used samples of the basal plate and placental bed specimens to compare trophoblast invasion in gestation-matched samples from normal pregnant patients and those with preeclampsia. These studies were complicated by the fact that the morphology of the placental bed changes during pregnancy, making it necessary to distin- guish between normal gestation-related changes and those that occurred as a result of the disease process. Taken together, this and our previous study (7) showed that during normal pregnancy a continuum of morphological changes takes place. Near the end of the first trimester, anchoring villi are connected to the uterus via extensive cell columns (data not shown; see reference 7). The cytokeratin-positive cytotrophoblasts that emanate from these columns lose their epithelial appearance and take on an elongated shape that is characteristic of highly migratory cells. This observation is consistent with the cell column as the source of the highly invasive subpopulation of cells that is found throughout the decidua, as well as the first third of the myometrium. At this stage, cytotrophoblast invasion of the blood vessels has not progressed beyond their decidual segments. As a result, the myometrial portions are intact.

The cell columns of the second trimester placenta $(22 \mathrm{wk}$; Fig. $1 \mathrm{~A}$ ) extend along the surface of the uterus and are thus shorter and wider than those of the first trimester. As evidenced by their elongated shape, cytokeratin-positive cytotrophoblasts are still migrating away from the column into the deeper portions of the uterus. At this stage cytotrophoblasts have replaced the endothelial lining and muscular coat of the arteries up to and including the portions that extend into the superficial myometrium (Fig. 1 B). By third trimester ( $36 \mathrm{wk}$ ), the columns are totally depleted of cells, although many cytotrophoblasts remain in the decidua (Fig. $1 C$ ). However, their shape is more rounded than in the first and second trimesters, commensurate with their diminished migratory capacity. Trophoblasts are still evident in the walls of blood vessels throughout the decidua and the superficial myometrium, although they are generally less numerous and are confined to the deeper portions of the vessel wall (Fig. 1 D).

As also described by other investigators (9-11), a much different situation exists in preeclampsia (Fig. 1, E-H). In all of the nine samples we studied, which ranged from late second trimester to term (25-37 wk), cytotrophoblast invasion was primarily confined to the superficial portions of the decidua. In general, the cytotrophoblasts retained their connections with one another instead of adopting the morphologic appearance associated with migratory cells. With regard to interactions with maternal cells, two patterns were seen. A striking feature of the three earliest-gestation samples $(25,27$, and $28 \mathrm{wk})$ was that many cytotrophoblasts remained in contact with the cell columns (Fig. $1 E$ ). In addition, no cytokeratin-positive cytotrophoblasts were seen in the deeper decidua and myometrium of these samples (Fig. $1 F$ ). In later-gestation ( $\geq 33 \mathrm{wk}$ ) samples, portions of cytotrophoblast columns remained in association with the anchoring villi, a feature never observed in the later stages of normal pregnancy. Most of the cytotrophoblasts that had lost their relationship to the cell columns were still clustered in the superficial decidua (Fig. $1 G$ ). Only a few cytokeratin-positive cytotrophoblasts with elongated shape were found in the myometrium (Fig. $1 \mathrm{H}$ ). None of the preeclamptic samples from any stage of gestation showed evidence of cytotrophoblast invasion of the uterine blood vessels, which were uniformly highly constricted (Fig. $1, F$ and $H$ ).

Integrin expression by cytotrophoblasts in the second and third trimesters of normal pregnancy. We recently showed that uterine invasion during the first trimester of pregnancy is consistently characterized by a dramatic change in the repertoire of adhesion receptors expressed by cytotrophoblasts as they dif- 


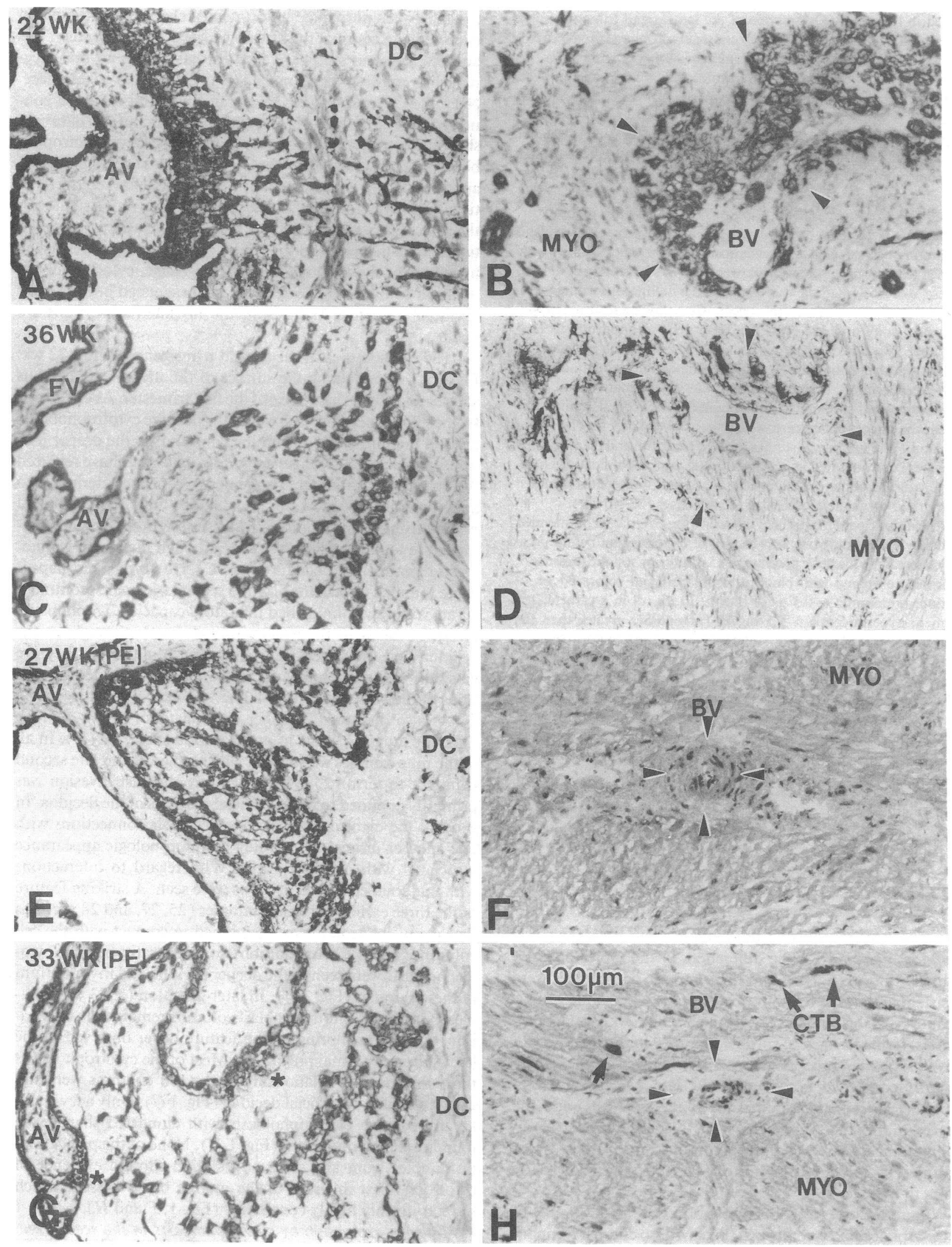

Figure 1. Anchoring villus formation and cytotrophoblast invasion in normal $(A-D)$ and preeclamptic $(E-H)$ pregnancy. Frozen sections of the maternal-fetal interface $(A, C, E$, and $G)$ and myometrium $(B, D, F$, and $H)$ were stained with anticytokeratin and peroxidase-conjugated sec- 
ferentiate along this pathway (7). Since the gestational age of the placental bed biopsies that were obtained from women with preeclampsia ranged from 25 to $37 \mathrm{wk}$, we first established the normal pattern of integrin expression by trophoblasts in the anchoring villi, basal plate, and uterus during the late second and the third trimesters of pregnancy.

During the late second trimester of pregnancy ( $22 \mathrm{wk}$; Fig. 2), modulation of trophoblast integrin expression during the invasion process was nearly identical to the pattern observed during the first trimester. Important hallmarks of this transition are the downregulation of the $\alpha 6$ and $\beta 4$ subunits and the upregulation of the $\alpha 5, \alpha 1$, and $\beta 1$ subunits as cells move from villus to uterus (7). The pattern of receptor regulation and the ligand specificities of these receptors are summarized in Table I. Specifically, second trimester villus cytotrophoblasts, which were anchored to their basement membrane, stained for $\alpha 6$ and $\beta 4$ integrin subunits. However, in the column an interesting transition was observed in which the staining for $\alpha 6 / \beta 4$ first became weak, and then finally absent (Fig. $2 C$ and $D$ ). In contrast, staining for the $\alpha 5$ and $\beta 1$ integrin subunits, which were not expressed by cytotrophoblasts anchored to their basement membranes, became strong in the column region (Fig. 2 $E$ and $F$ ). Staining for the $\alpha 1$ subunit, which was also absent on cytotrophoblasts in contact with the basement membrane, was likewise detected in the column (Fig. $2 G$ ), but in a region distal to the area where $\alpha 5$ staining was first detected. Within the uterine wall cytotrophoblasts continued to stain with antibodies that recognized the $\alpha 5, \alpha 1$, and $\beta 1$ integrins. In this location staining for $\alpha 6$ and $\beta 4$ was absent (Figs. $2 C$ and $D$ ). However, the staining patterns of the first and the second trimester placental bed specimens were not entirely identical. The major difference was that during the first trimester the $\alpha 3$ subunit was expressed only by some villus cytotrophoblasts and was absent on column and placental bed cytotrophoblasts. In contrast, staining for this integrin was uniform on second trimester villus cytotrophoblasts and was also detected on column and placental bed cytotrophoblasts (Fig. $2 \mathrm{H}$ ).

By the third trimester, cell columns are no longer visible ( see Fig. $1 C$ ), so the anchoring villus transition zone could not be evaluated. Cytotrophoblasts within the uterine wall, like those of the second trimester placental bed, stained for $\alpha 5, \alpha 1$, $\alpha 3$, and $\beta 1$ integrins. However, there was one notable difference between the late second trimester and term cytotrophoblast staining patterns. The expression of the $\alpha 6$ and $\beta 4$ subunits, which together compose the major integrin receptor expressed by first trimester villus cytotrophoblasts, was differentially regulated. Staining for the $\alpha 6$ subunit was once again prominent (Fig. $3 C$ ), whereas staining for $\beta 4$ was not detected (Fig. $3 \mathrm{D}$ ).

In summary, in tissues from normal pregnancies we ob- served two patterns of cytotrophoblast integrin switching. One transition took place in the cell column of the anchoring villus and was characterized by a downregulation of $\alpha 6 / \beta 4$ and an upregulation first of $\alpha 5 / \beta 1$ and then of $\alpha 1 / \beta 1$. The second transition occurred as a function of gestational age. From the second trimester onward, $\alpha 3$ was expressed by cytotrophoblasts in all locations. At term, placental bed cytotrophoblasts expressed $\alpha 6$, but not $\beta 4$. The consistency with which these staining patterns were observed in normal pregnancy allowed us to determine next whether abnormal integrin expression was associated with the abnormally shallow invasion that is characteristic of preeclampsia.

Trophoblast expression of integrin cell-matrix receptors is altered in preeclampsia. Cytotrophoblast expression of two of the integrin receptors, $\alpha 6 / \beta 4$ and $\alpha 1 / \beta 1$, was dramatically and consistently disregulated in preeclampsia and was the same whether the tissue samples were obtained by cesarean section or after vaginal delivery (summarized in Table I and Figs. 4 and 5). Cytotrophoblasts in floating villi from preeclamptic pregnancies expressed primarily $\alpha 6 / \beta 4$, a pattern that was identical to that observed during normal pregnancy (data not shown). However, instead of becoming downregulated in the anchoring villus column and in the uterine wall, as occurs in normal pregnancy, this receptor was strongly expressed by cytotrophoblasts within the placental beds of preeclamptic patients. In the late second trimester samples (e.g., 25 wk; Fig. $4 A$ ), cytokeratin staining again demonstrated that cytotrophoblast invasion is confined to a narrow portion of the superficial decidua. Cytotrophoblasts in this location stained strongly for both $\alpha 6$ (Fig. $4 \mathrm{~B}$ ) and $\beta 4$ (data not shown), a pattern that was never observed at similar stages of normal pregnancy (Fig. $2 D$ and $E$ ). Cytotrophoblast staining for both $\alpha 6$ and $\beta 4$ was also observed in later-gestation placental bed specimens from preeclamptic patients ( $33 \mathrm{wk}$; Fig. $4 D$ and $F$ ). At this stage of normal pregnancy, cytotrophoblasts in the placental bed stain for $\alpha 6$ but not $\beta 4$ (Fig. $3 D$ and $E$ ). Thus, placental bed cytotrophoblasts from preeclamptic patients stain for $\alpha 6$ at a time during the second trimester when expression of this integrin subunit is normally not observed. Furthermore, cytotrophoblast staining for $\beta 4$, which is never detected on cells within the uterine wall, was prominent on cytotrophoblasts of the preeclamptic placental bed.

Cytotrophoblasts in the uterine wall in preeclampsia also failed to upregulate the expression of $\alpha 1 / \beta 1$ (Fig. $5 B$ ). In four of the nine preeclamptic patients, no $\alpha 1$ staining was detected. In the remaining samples, a few $(<5 \%)$ cytotrophoblasts exhibited barely detectable levels of staining. Normally, this receptor is consistently expressed by placental bed cytotrophoblasts at all stages of gestation (Figs. $2 G$ and $3 G$ and reference 7). As discussed in more detail below, staining is particularly strong in

ondary antibodies. During the second trimester (22 wk) cytokeratin-positive cytotrophoblasts with elongated shape emanate $(A)$ from the anchoring villus $(A V)$ columns and are found throughout the decidua $(D C),(B)$ from the adjacent third of the myometrium and lining the blood vessels $(B V)$. By $36 \mathrm{wk}(C)$, cell columns are absent, although rounded cytotrophoblasts are still visible in the decidua and $(D)$ in reduced numbers in the walls of blood vessels. $F V$, floating villus. In preeclampsia $(P E)$, two patterns are seen. In the first $(E$ and $F ; 25-28$ wk), cytotrophoblasts retain their connections with the cell columns and fail to invade the decidua and myometrium. There is no evidence of cytotrophoblast invasion of the blood vessels, which remain uniformly constricted. In the second pattern ( $G$ and $H$; 33-37 wk), some cytotrophoblasts $(C T B)$ lose their attachments to the cell columns $\left({ }^{*}\right)$, which, in contrast to the same stage of normal pregnancy, are still a prominent feature of the maternal-fetal interface. $(H)$ Also in the second pattern a few cytokeratin-positive cytotrophoblasts penetrate the myometrium, but not the highly constricted blood vessels. Arrowheads mark perimeters of blood vessels; arrows point to cytotrophoblasts. 

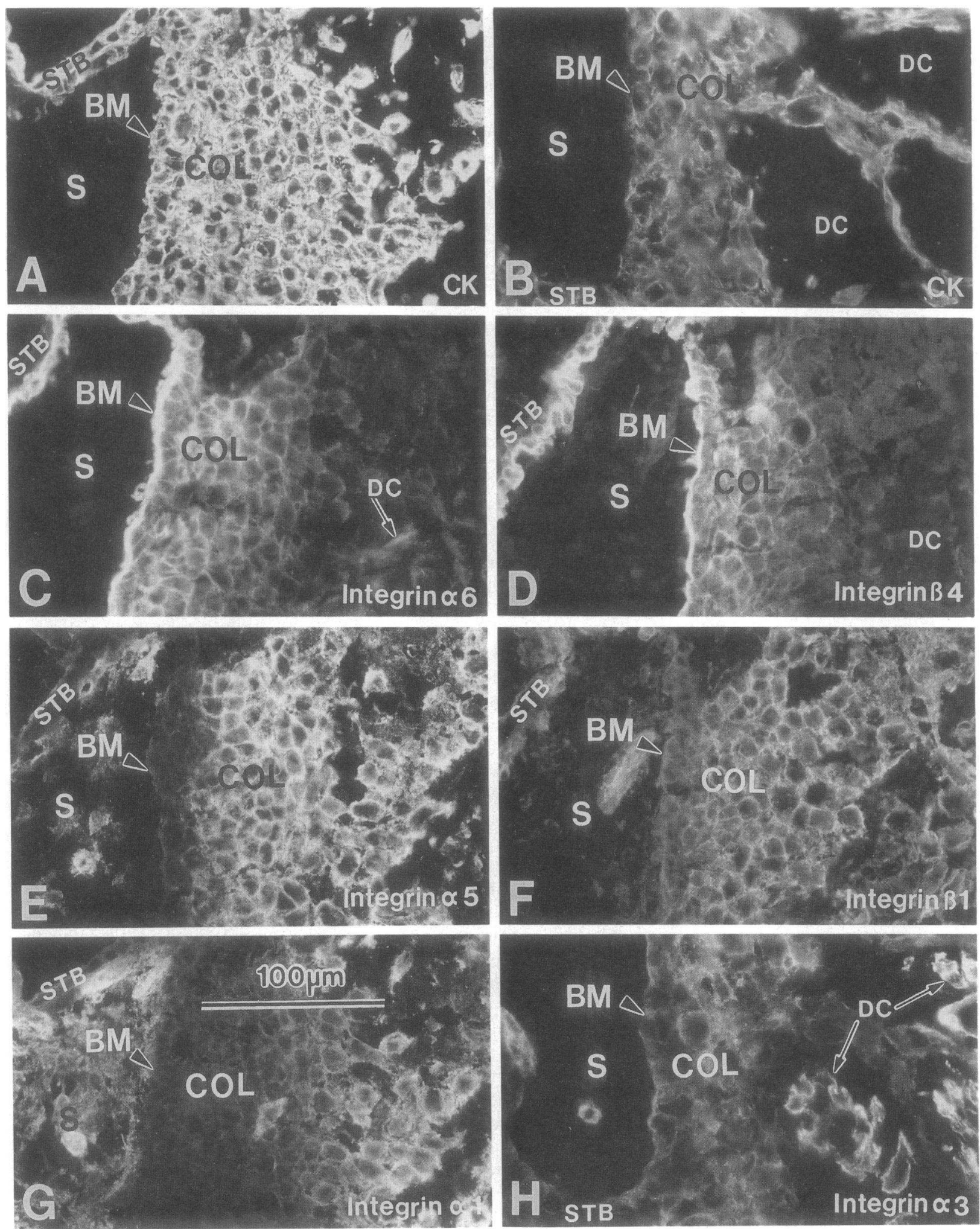

Figure 2. Integrin subunits expressed by cytotrophoblasts in second trimester ( $22 \mathrm{wk}$ ) anchoring villi from normal pregnancy. $A, C, D, E, F$, and $G$ are serial sections, as are $B$ and $H . A$ and $B$ are the cytokeratin-positive controls for these two series. $(C)$ Anti- $\alpha 6$ and $(D)$ anti- $\beta 4$ strongly stained cytotrophoblasts that are still in contact with their basement membrane $(B M)$, as well as those in the proximal column $(C O L)$. As the column progressed toward the uterus, staining decreased, becoming undetectable as the cells contacted the decidua $(D C)$. $C K$, cytokeratin; $S$, stroma; $S T B$, syncytiotrophoblast. Conversely, $(E)$ anti- $\alpha 5,(F)$ anti- $\beta 1$, and $(G)$ anti- $\alpha 1$ did not stain cytotrophoblasts that were in contact with their own basement membrane. Expression of $\alpha 5$ and $\beta 1$ was first observed in the proximal column and that of $\alpha 1$ in the more distal column. All three integrin subunits continued to be expressed by cytotrophoblasts in the uterine wall. $(H)$ The $\alpha 3$ subunit, which is variably expressed by first-trimester cytotrophoblasts, is detected on second-trimester villus, column, and placental bed cytotrophoblasts. 


\begin{tabular}{clll}
\hline & & \multicolumn{2}{c}{ Change from villus to placental bed } \\
\cline { 3 - 4 } Integrin receptor & ECM ligands & Normal pregnancy & Preeclampsia \\
\hline$\alpha 6 / \beta 4$ & Basement membrane ligand (Ln?) & Downregulated & Unchanged* $^{*}$ \\
$\alpha 5 / \beta 1$ & Fn & Upregulated & Upregulated \\
$\alpha 3 / \beta 1$ & Ln, Fn, Col & Uniformly expressed & Uniformly expressed \\
$\alpha 1 / \beta 1$ & Ln, Col IV, Col I & Upregulated & Not expressed \\
& & & $($ weakly expressed) \\
& & &
\end{tabular}

\footnotetext{
* The $\beta 4$ subunit is absent on placental bed cytotrophoblasts throughout gestation. Staining for $\alpha 6$ is absent on these cells through the second trimester but is reexpressed at term. In preeclampsia $\alpha 6 / \beta 4$ is expressed by placental bed cytotrophoblasts in both the second and third trimesters. ₹In $5 / 9$ preeclamptic samples the majority $(>95 \%)$ of placental bed cytotrophoblasts did not stain with anti- $\alpha 1$ antibody, but a few weakly positive cytotrophoblasts were detected near the surface of the decidua.
}

association with cytotrophoblasts that have invaded blood vessels.

In contrast, cytotrophoblast expression of other integrins in preeclampsia was the same as in normal pregnancy. Cytotrophoblast staining for the $\alpha 3$ (Fig. $5 F$ ), $\alpha 5$ (Fig. $5 C$ ), and $\beta 1$ (Fig. $4 F$ ) subunits appeared at the appropriate time in the anchoring villus column and was maintained at normal levels in the placental bed. Since, as described in the preceding paragraph, $\alpha 6$ staining was also detected, it is likely that cytotrophoblasts in the uterine wall express $\alpha 6 / \beta 1$ complexes at least as early as the late second trimester, a situation that does not exist in normal pregnancy until term.

We also examined integrin expression during blood vessel invasion in normal pregnancy and in preeclampsia (Fig. 6). The presence of cytotrophoblasts in the walls of blood vessels was associated with particularly strong staining of the fetal cells for the $\alpha 1$ subunit during the first (data not shown) and second trimesters of normal pregnancy (Fig. $6 \mathrm{~B}$ ). At this stage cytokeratin-positive cytotrophoblast clusters were also found plugging the vessel lumen (Fig. $6 C$ ). These cells stained intensely with anti- $\alpha 1$. By term, the majority of cytotrophoblasts that remained in association with blood vessels were found deep within the wall (Fig. $6 \mathrm{E}$ ). In this location they continued to express $\alpha 1$ (Fig. $6 F$ ). As described by other investigators, preeclampsia is characterized by a failure of the cytotrophoblast cells to invade the maternal blood vessels. We also found this to be the case. No cytokeratin-positive cells were found in association with any of the maternal blood vessels, which remained highly constricted (Fig. $6 \mathrm{G}$ ). The only $\alpha 1$ staining detected was in association with maternal cells (Fig. $6 \mathrm{H}$ ).

Trophoblast expression of extracellular matrix molecules is not altered in preeclampsia. Finally, we examined the expression of extracellular matrix molecules in the floating and anchoring villi and in the placental beds of normal and preeclamptic patients (data not shown). Unlike their integrin receptors, the pattern of expression of this class of molecules was nearly the same in both groups and very similar to that we had previously described during the first trimester of normal pregnancy (7). Therefore, unless otherwise noted, the following description of the expression of extracellular matrix molecules applies to second and third trimester placental samples obtained from both normal and preeclamptic pregnancies. Briefly, cytotrophoblasts that remained in the floating villi were anchored to a basement membrane in which all the known isoforms of la-
$\operatorname{minin}(\mathrm{Ln})^{1}$ were detected: the $\mathrm{Ln} \mathrm{B} 2$ chain, $\mathrm{Ln} \mathrm{M}$ (merosin), $\mathrm{Ln} A$ chain, and $\mathrm{Ln} \mathrm{S}$ as well as the related $\mathrm{Ln} \mathrm{B} 1$ chain. All these $\mathrm{Ln}$ isoforms were also detected in the villus stroma, as were fibronectin that includes the EIIIA and EIIIB alternatively spliced domains $\left(\mathrm{A}^{+} \mathrm{B}^{+} \mathrm{Fn}\right)$ and collagen type IV ( Col IV). Fn staining of the trophoblast basement membrane was weak, whereas that of Col IV was intense.

Samples that were obtained early enough in gestation to contain cell columns showed the striking alteration in the staining patterns of cytotrophoblast-associated extracellular matrix molecules we previously described (7). In the proximal portion of the column, $\mathrm{A}^{+} \mathrm{B}^{+} \mathrm{Fn}$ and $\mathrm{Col}$ IV were not detected and the distribution of individual $\mathrm{Ln}$ subunits differed. $\mathrm{Ln} \mathrm{M}$ and Ln S were not detected on column cytotrophoblasts, whereas the $\mathrm{Ln} A$ and $\mathrm{B} 1$ subunits and $\mathrm{Ln} \mathrm{B} 2$ were present. In the distal portion of the column, there was a sharp increase in the level of staining of both $\mathrm{A}^{+} \mathrm{B}^{+} \mathrm{Fn}$ and Col IV.

Cytotrophoblasts in the placental beds of normal patients stained weakly and variably for $\mathrm{Ln} A$ and B1, but expression of Ln B2 was strong. There was a tendency for the cytotrophoblast-associated staining of $\operatorname{Ln} A$ and $\operatorname{Ln} B 2$ to be stronger in the placental beds of preeclamptic patients. In this location cytotrophoblasts from both normal and preeclamptic patients expressed variable levels of $\mathrm{A}^{+} \mathrm{B}^{+} \mathrm{Fn}$ and $\mathrm{Col}$ IV. This was in contrast to our previous observation that first trimester placental bed cytotrophoblasts failed to stain for either Fn or Col IV (7) and suggested an age-related change in the pattern of expression of these molecules.

\section{Discussion}

The results of this study confirm previous observations (9-12) concerning the abnormally shallow trophoblast invasion of the uterus that is associated with preeclampsia. Using placental bed biopsy specimens from preeclamptic patients, we observed two distinct patterns of cytotrophoblast interactions with maternal cells that were characteristic of either the earlier (25-28 wk) or the later-gestation ( $\geq 33 \mathrm{wk}$ ) samples. Because samples of the placental bed are obtained only at cesarean section, we were unable to distinguish between two possible explanations

1. Abbreviations used in this paper: Col IV, collagen type IV; Fn, fibronectin; Ln, laminin. 

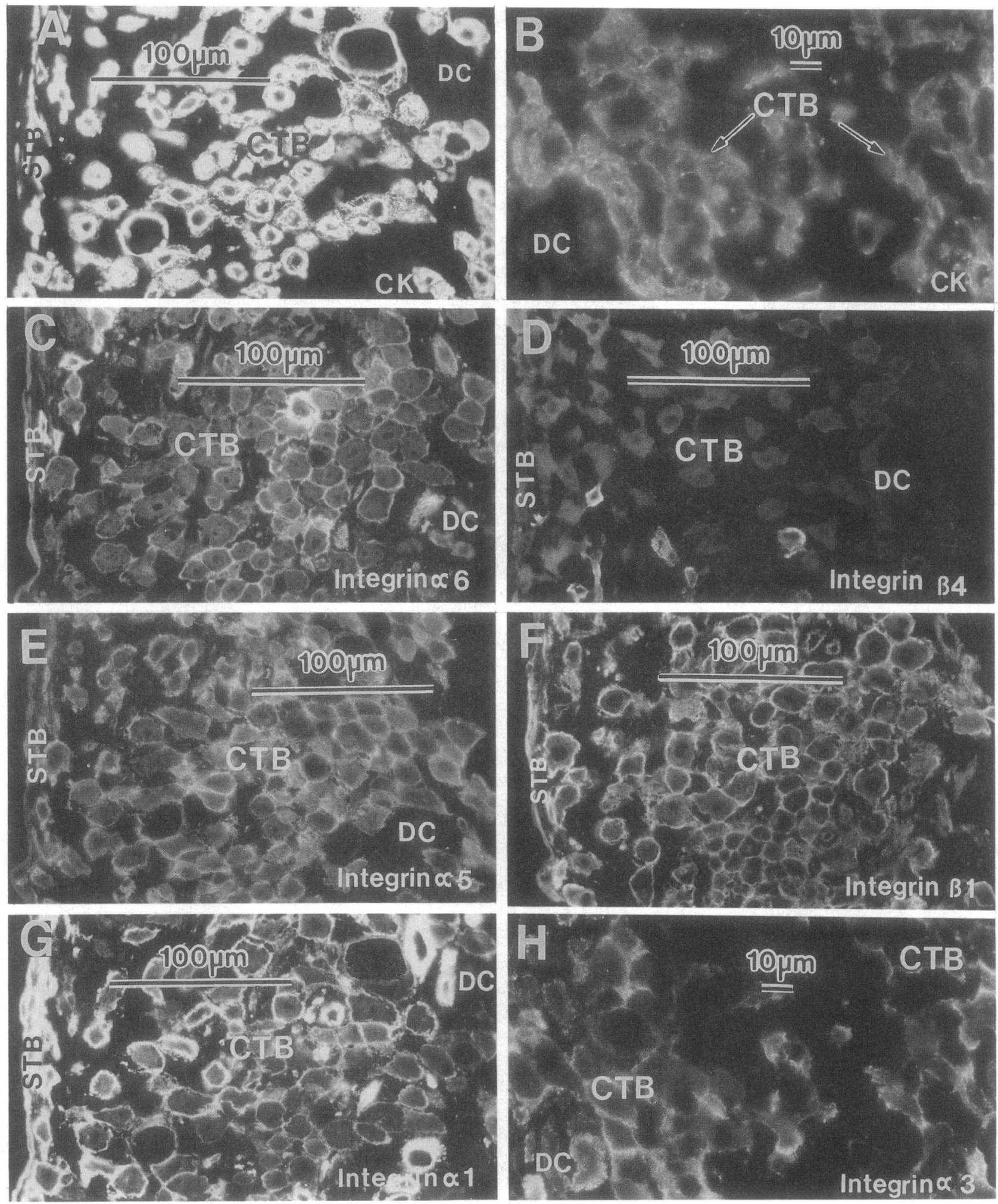

Figure 3. Integrin subunits expressed by cytotrophoblasts within the uterine wall near the end of normal pregnancy ( 36 wk). $A, C, D, E, F$, and $G$ are serial sections, as are $B$ and $H$. $A$ and $B$ are the cytokeratin-positive controls for these two series. ( $C$ ) At term, integrin $\alpha 6$ expression was again detected, $(D)$ although cytotrophoblast staining for $\beta 4$ was not. Cytotrophoblasts in the uterine wall continued to express $(E) \alpha 5,(F) \beta 1$, (G) $\alpha 1$, and $(H) \alpha 3$ (abbreviations as in Fig. 2.).

for this observation. The more restricted invasion seen in the earlier samples could be associated with a more severe form of the disease that required delivery of the baby before the 30th week of gestation. Alternatively, a continuum of invasion, al- though limited in scope, may also occur in preeclampsia. If this is the case, then the earlier-gestation samples would lie at the beginning of this continuum and those from later gestation at the end. 

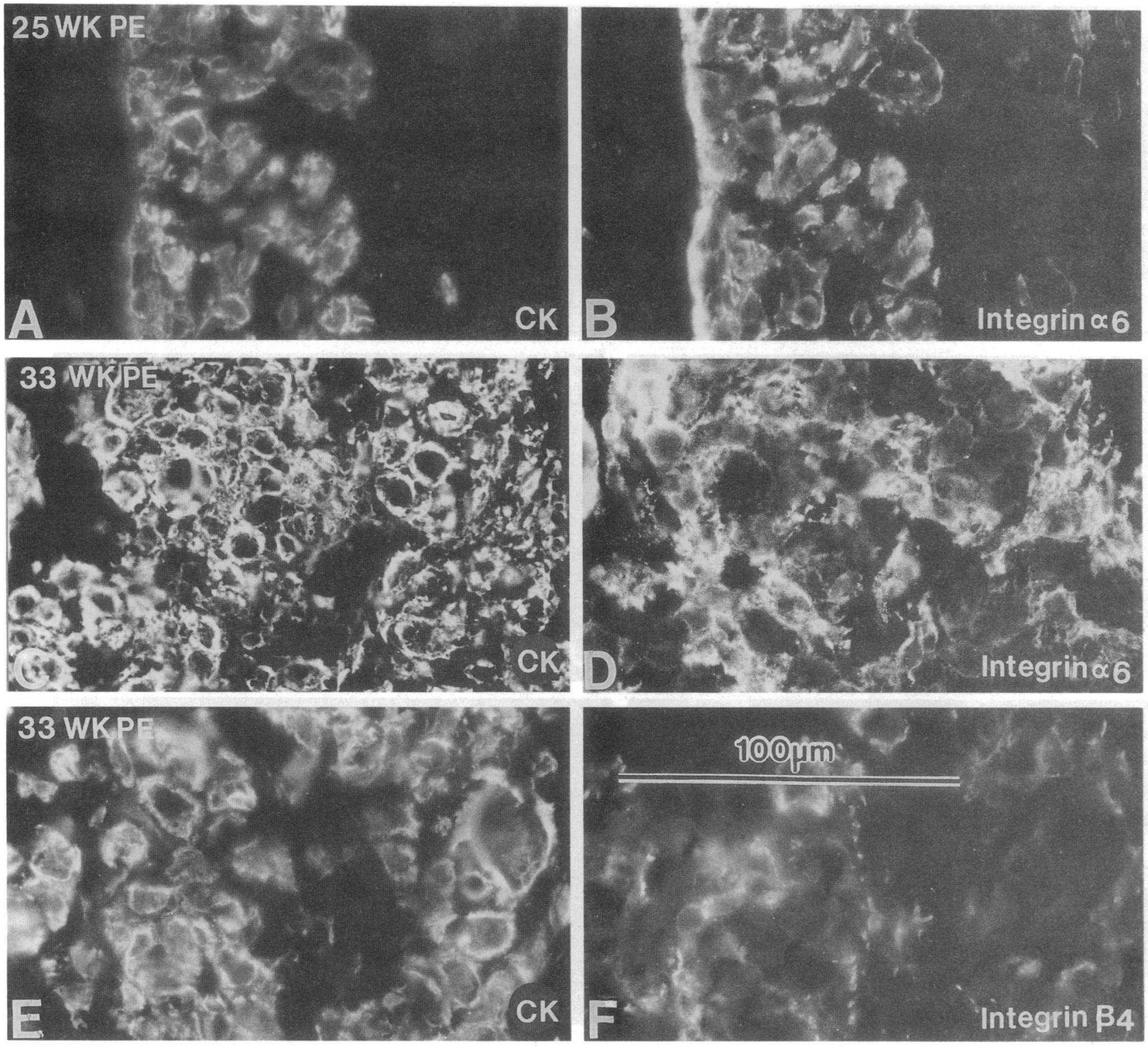

Figure 4. Placental bed cytotrophoblast staining for $\alpha 6$ and $\beta 4$ integrins is abnormal in preeclampsia. The sections shown in $A$ and $B, C$ and $D$, and $E$ and $F$ were double-stained with anticytokeratin $(C K)$ and antiintegrins. $(B)$ At 25 wk gestation, cytotrophoblasts in the placental bed of a preeclamptic patient exhibited strong staining for both $\alpha 6$ and (not shown) $\beta 4$. This is in contrast to the pattern shown at 22 wks of normal pregnancy (Fig. $2 C$ and $D$ ), in which cytotrophoblasts in the placental bed did not stain for either $\alpha 6$ or $\beta 4$. An abnormal staining pattern was also found in later-gestation biopsies from preeclamptic patients. For example, at 33 wk placental bed cytotrophoblast staining for $(D) \alpha 6$ and ( $F) \beta 4$ was also strong.

We expanded the scope of these morphological observations to show that cytotrophoblast expression of cell-extracellular matrix receptors (integrins), potentially important mediators of the invasion process, is abnormal in preeclampsia. The results of this and our previous study (7) showed that cytotrophoblast progression through the cell column is normally characterized by downregulation of the integrin $\alpha 6 / \beta 4$, which may be a receptor for laminin (20). Conversely, cells in the column upregulate their production of the major fibronectin receptor $(\alpha 5 / \beta 1)$. Within the uterine wall, a final transition is observed in which the expression of a laminin-collagen receptor $(\alpha 1 / \beta 1)$ is upregulated. This study showed that gestation-related alterations also occur. Comparing integrin expression by placental bed cytotrophoblasts during all three trimesters of pregnancy, we observed that $\alpha 3$ expression is detected from the second trimester onward, and $\alpha 6$ but not $\beta 4$ expression reappears at term.

In preeclamptic women, the expression of only a subset of these cell-cell and cell-matrix adhesion molecules is regulated normally. Staining for the $\alpha 3, \alpha 5$, and $\beta 1$ subunits was similar to that observed in samples from age-matched control patients. In contrast, the pattern of expression of the other cell adhesion molecules was very different. The $\alpha 6$ and $\beta 4$ subunits continued to be strongly expressed within the uterine wall, and $\alpha 1 / \beta 1$ was either not upregulated or only weakly expressed by a small proportion $(<5 \%)$ of cytotrophoblasts within the uterine wall. These observations suggest that in preeclampsia, cytotrophoblasts that invade the uterus retain cell adhesion molecules that 

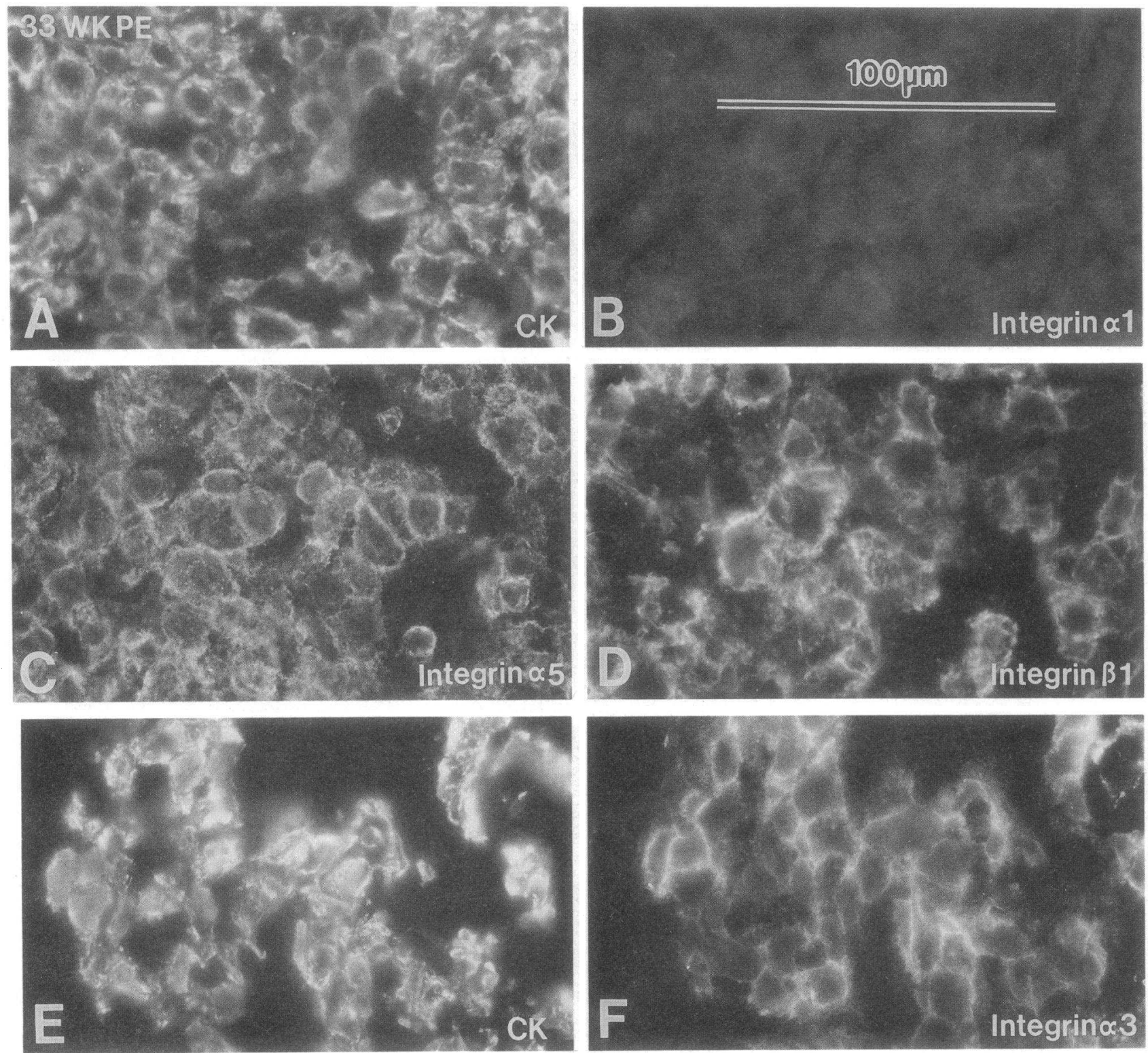

Figure 5. Placental bed cytotrophoblast staining for integrin $\alpha 1$ is abnormal in preeclampsia ( $33 \mathrm{wk}$ ). $A$ and $B$, and $E$ and $F$ were double-stained with anticytokeratin $(C K)$ and antiintegrins. $A, B$, and $C$ are serial sections. $(B)$ Integrin $\alpha 1$ staining was either not detected or weakly positive on cytotrophoblasts in the placental beds of preeclamptic patients at all stages of gestation studies (25-37 wk). This is in contrast to the pattern during the second (Fig. $2 G$ ) and third (Fig. $3 G$ ) trimesters of normal pregnancy in which cytotrophoblasts in the placental bed stained strongly for $\alpha 1$. However, as in normal pregnancy, cytotrophoblasts in the uterine wall of preeclamptic patients expressed $(C) \alpha 5,(D) \beta 1$, and $(F) \alpha 3$.

under normal conditions are expressed only by villus stem cells and cytotrophoblasts in the proximal region of the cell columns. In addition, they fail to upregulate the expression of one integrin receptor that in normal pregnancy is expressed only by cytotrophoblasts that have invaded the uterine wall. The observed disregulation of integrin expression is in striking contrast to that of the extracellular matrix ligands for these receptors, which is normal.

The results of this study correlate well with those we have recently obtained using an in vitro model of cytotrophoblast invasion (21) and function-perturbing antiintegrin antibodies to assess the relative contribution of individual cell-matrix receptors to this process. Antibodies to the $\alpha 5$ integrin subunit accelerated cytotrophoblast invasion by over twofold, and anti- bodies to $\alpha 1$ inhibited cytotrophoblast invasion (reference 14; Damsky, C. H., and S. J. Fisher, manuscript in preparation). Thus, it is likely that cytotrophoblast interactions with fibronectin constrain cytotrophoblast invasion, whereas interactions with laminin via $\alpha 1 / \beta 1$ facilitate this process. Our observation that $\alpha 5 / \beta 1$, but not $\alpha 1 / \beta 1$, is upregulated in invasive cytotrophoblasts present in placental bed biopsies of preeclamptic patients therefore indicates that the adhesion phenotype of these cells is not optimal for invasion. Although antibodies to $\alpha 6 \mathrm{did}$ not enhance invasion, the preferential association of $\alpha 6 / \beta 4$ with hemidesmosomes in stable epithelia (22) suggests a role in mediating similar interactions involving cytotrophoblasts. Thus, the functions of these cell-adhesion molecules suggest that failure of invasive cytotrophoblasts to downregulate adhe- 

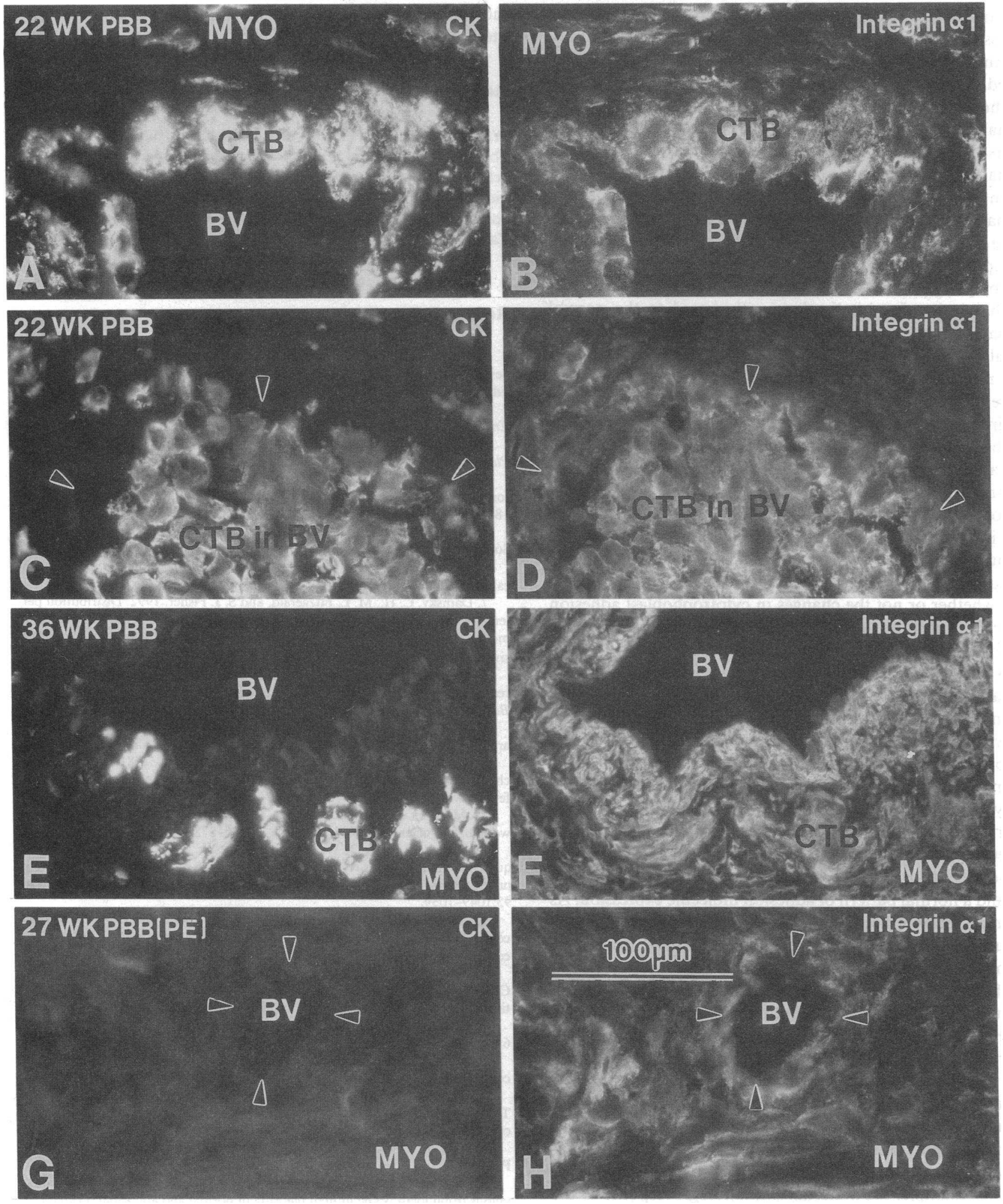

Figure 6. Integrin subunits expressed by cytotrophoblasts that penetrate uterine blood vessels in normal pregnancy $(A-F)$ and absence of vessel penetration in preeclampsia $(G$ and $H) . A$ and $B, C$ and $D, E$ and $F$, and $G$ and $H$ were double-stained with anti-cytokeratin $(C K)$ and anti- $\alpha 1$. By the end of the second trimester, cytokeratin-positive cytotrophoblasts $(C T B)(A)$ replaced the endothelial lining of many of the blood vessels $(B V)$ and $(C)$ formed plugs in others. Cytotrophoblasts that $(B)$ line vessel walls and $(D)$ lodge in their lumina stain very strongly for integrin $\alpha 1$. By $36 \mathrm{wk}(E)$ only a few cytokeratin-positive cytotrophoblasts remain in association with the blood vessels, $(F)$ but cells in this location continue to stain with anti- $\alpha 1$. ( $G$ and $H$ ) In preeclampsia $(P E)$, no cytokeratin-positive cytotrophoblasts invade the blood vessels, and $\alpha 1$ staining is confined to the resident maternal cells. $P B B$, placental bed biopsy; arrowheads indicate vessel lumen. 
sion mechanisms used in the villus and to upregulate those expressed in the uterine wall could lead to the shallow trophoblast invasion that is characteristic of the preeclamptic placental bed.

The observation that invasive cytotrophoblasts exhibit abnormal antigen expression in preeclampsia offers insight into factors that may play important roles in both normal and abnormal trophoblast invasion. The results of our study of the placental bed during the second and third trimesters of normal pregnancy suggest that one of the integrin $\alpha$ subunits $(\alpha 6)$, whose expression, together with $\beta 4$, is disregulated in preeclampsia, is strongly reexpressed with a different $\beta$ subunit $(\beta 1)$ by placental bed cytotrophoblasts at term. This observation has interesting implications with regard to the normal mechanisms by which cytotrophoblast invasion is limited both spatially and temporally. Disregulation of the expression of these molecules in preeclampsia suggests that cytotrophoblast differentiation is abnormal in this disease. The data we have acquired thus far do not allow us to distinguish between the possibilities that cytotrophoblasts either fail to fully differentiate along the invasive pathway or switch prematurely to a third-trimester phenotype. However, the fact that the expression of the laminin-collagen receptor $(\alpha 1 / \beta 1)$ is not upregulated in preeclampsia is evidence that the former, rather than the latter, hypothesis may be the case.

Currently, we do not have enough information to determine whether or not the changes in cytotrophoblast adhesion molecule expression are specific to preeclampsia. It is possible that this abnormal pattern characterizes other diseases of pregnancy that are associated with shallow trophoblast invasion, such as intrauterine growth retardation (12). However, we did obtain placental bed biopsies from a woman whose first pregnancy was complicated by severe preeclampsia and whose second pregnancy was complicated by intrauterine growth retardation. The placental bed biopsy specimen from her first pregnancy ( $28 \mathrm{wk}$ ) showed the shallow invasion and abnormal pattern of adhesion molecule expression that are characteristic of preeclampsia. In contrast, the placental bed biopsy from the second pregnancy ( $36 \mathrm{wk}$ ) showed both a normal level of invasion and a normal pattern of adhesion molecule expression. As placental bed biopsies are obtained from more patients with intrauterine growth retardation, we will determine whether or not the changes in adhesion molecule expression are specific to preeclampsia or also associated with other disorders.

An abnormality of the placenta has been implicated as the underlying cause of preeclampsia $(2,3)$. Our observations are in agreement with this theory and suggest the interesting possibility that this disease is the result of abnormal cytotrophoblast differentiation such that they do not display the optimum adhesion phenotype for invasion. Currently, the factors that control this differentiation process are not well understood; by analogy with other systems, they are likely to be extremely complex. For example, the maternal immunologic response to the fetus could play an important role (23). Nevertheless, it is likely that abnormal expression of regulatory molecules by either the cytotrophoblasts or the maternal cells with which they come into contact is responsible for the abnormalities in adhesion-related antigen expression we observed. Thus, the failure of cytotrophoblasts to switch their adhesion molecule phenotype in preeclampsia could tip the delicate balance of molecules that normally permit cytotrophoblast invasion in favor of those that restrain this process, with the net effect of shallow uterine invasion.

\section{Acknowledgments}

We thank Ms. Jean Perry for her invaluable assistance with regard to obtaining the placental bed biopsies and Ms. Evangeline Leash for excellent editorial assistance. The following investigators generously provided antibodies for this study: Drs. Steven Kennel, Vera Morhenn, Joshua Sanes, Arnoud Sonnenberg, Elizabeth Wayner, and Luciano Zardi.

This work was supported by HD24180 and HD22210.

\section{References}

1. Roberts, J. M., R. N. Taylor, S. A. Friedman, and A. Goldfien. 1993. New developments in pre-eclampsia. In Fetal Medical Review. W. Dunlop, editor. Edward Arnold Publishers, London. In press.

2. Redman, C. W. G. 1990. Platelets and the beginnings of preeclampsia. $N$. Engl. J. Med. 323:478-480.

3. Redman, C. W. G. 1991. Current topic: pre-eclampsia and the placenta Placenta. 12:301-308.

4. Chun, D., C. Braga, C. Chow, and L. Lok. 1964. Clinical observations on some aspects of hydatidiform moles. J. Obstet. Gynecol. Br. Commonw. 71:180 184.

5. Boyd, J. D., and W. J. Hamilton. 1970. The Human Placenta. Heffer and Sons, Cambridge, UK. 365 pp.

6. Pijnenborg, R. 1990. Trophoblast invasion and placentation: morphological aspects. Troph. Res. 4:33-50.

7. Damsky, C. H., M. L. Fitzgerald, and S. J. Fisher. 1992. Distribution patterns of extracellular matrix components and adhesion receptors are intricately modulated during first trimester cytotrophoblast differentiation along the invasive pathway, in vivo. J. Clin. Invest. 89:210-222.

8. Brosens, I., W. B. Robertson, and H. G. Dixon. 1967. The physiological response of the vessels of the placental bed to normal pregnancy. J. Pathol. Bacteriol. 93:569-579.

9. Brosens, I., W. B. Robertson, and H. G. Dixon. 1972. The role of the spiral arteries in the pathogenesis of pre-eclampsia. Obstet. Gynecol. Annu. 1:177-191.

10. Gerretsen, G., H. J. Huisjes, and J. D. Elema. 1981. Morphological changes of the spiral arteries in the placental bed in relation to preeclampsia and fetal growth retardation. Br. J. Obstet. Gynaecol. 88:876-881.

11. Moodley, J., and R. Ramsaroop. 1989. Placental bed morphology in black women with eclampsia. S. Afr. Med. J. 75:376-378.

12. Khong, T. Y., F. De Wolf, W. B. Robertson, and I. Brosens. 1986. Inadequate maternal vascular response to placentation in pregnancies complicated by pre-eclampsia and by small-for-gestational-age infants. Br. J. Obstet. Gynaecol. 93:1049-1059.

13. Lunell, N.-O., L. E. Nylund, R. Lewander, and B. Sarby. 1982. Uteroplacental blood flow in pre-eclampsia. Measurements with indium-113m and a computer-linked gamma camera. Clin. Exp. Hypertens. B1:105.

14. Librach, C., S. J. Fisher, M. L. Fitzgerald, and C. H. Damsky. 1991 Cytotrophoblast-fibronectin and cytotrophoblast-laminin interactions have distinct roles in cytotrophoblast invasion. J. Cell Biol. 115:6a. (Abstr.)

15. Chesley, L. 1985. Diagnostic criteria for preeclampsia. Obstet. Gynecol. 65:423-425.

16. Lind, T., K. A. Godfrey, and H. Otun. 1984. Changes in serum uric acid concentrations during normal pregnancy. Br. J. Obstet. Gynaecol. 91:128-132.

17. Fisher, S. J., T-Y. Cui, L. Zhang, L. Hartman, K. Grahl, G-Y. Zhang, J. Tarpey, and C. H. Damsky. 1989. Adhesive and invasive interactions of human cytotrophoblast cells in vitro. J. Cell Biol. 109:891-902.

18. Fox, H. 1979. The placenta in maternal disorders. In Pathology of the Placenta. W. B. Saunders, London. 214-222.

19. Arnholdt, H., F. Meisel, K. Fandrey, and U. Lohrs. 1991. Proliferation of villous trophoblast of the human placenta in normal and abnormal pregnancies. Virchows Arch. Cell. Pathol. 60:365-372.

20. Lee, E. C., M. M. Lotz, G. D. Steele, Jr., and A. M. Mercurio. 1992. The integrin $\alpha 6 / \beta 4$ is a laminin receptor. J. Cell Biol. 117:671-678.

21. Librach, C., Z. Werb, M. L. Fitzgerald, K. Chiu, N. Corwin, R. Esteves, R. Galardy, C. H. Damsky, and S. J. Fisher. 1991b. 92 Type IV collagenase mediates invasion of human cytotrophoblasts. J. Cell Biol. 113:437-449.

22. Quaranta, V., and J. C. R. Jones. 1991. The internal affairs of an integrin. Trends Cell Biol. 1:2-4.

23. Redman, C. W. 1981. Immunological factors in the pathogenesis of preeclampsia. Contrib. Nephrol. 25:120-127. 